\title{
Ciphertext only Reconstruction of Stream Ciphers Based on Combination Generators
}

\author{
Anne Canteaut ${ }^{1}$ and Eric Filiol ${ }^{1,2}$ \\ 1 INRIA, projet CODES, Domaine de Voluceau \\ 78153 Le Chesnay Cedex, FRANCE \\ \{Anne.Canteaut,Eric.Filiol\}@inria.fr \\ 2 Ecoles militaires de Saint-Cyr Coëtquidan, \\ DGER/CRECSC/DSI, 56381 Guer Cedex, FRANCE \\ efiliol@mailhost.esm-stcyr.terre.defense.gouv.fr
}

\begin{abstract}
This paper presents an operational reconstruction technique of most stream ciphers. We primarily expose it for key-stream generators which consist of several linear feedback shift registers combined by a nonlinear Boolean function. It is shown how to completely recover the different feedback polynomials and the combining function, when the algorithm is totally unknown. This attack only requires the knowledge of some ciphertexts, which may be generated from different secret keys. Estimates of necessary ciphertext length and experimental results are detailed.
\end{abstract}

Keywords: stream cipher, Boolean function, correlation, linear feedback shift register, ciphertext only reconstruction

\section{Introduction}

Stream ciphers are an important class of cipher systems. They are widely used by the world's militaries and governmental offices. They also are very often used in industrial encryption products. The success of stream ciphers comes from the fact that they are very easy to build: they need only few logic gates in VLSI circuitry. They are therefore particularly appropriate to embedded systems (satellites for example) or to the systems for which maintenance is either impossible or very difficult. Moreover, their use is particularly well-suited when errors may occur during the transmission because they avoid error propagation.

In a binary additive stream cipher, the ciphertext is obtained by adding bitwise the plaintext to a pseudo-random sequence called the running-key (or the key-stream). The running-key is produced by a pseudo-random generator whose initialization is the secret key shared by the users. Most practical designs of pseudo-random generators center around linear feedback shift registers (LFSRs) combined by a nonlinear Boolean function. Different variants can actually be found: clock-controlled systems, filter generators, multiplexed systems... 13. We here focus on the most common class of combination generators depicted in Figure 1. 


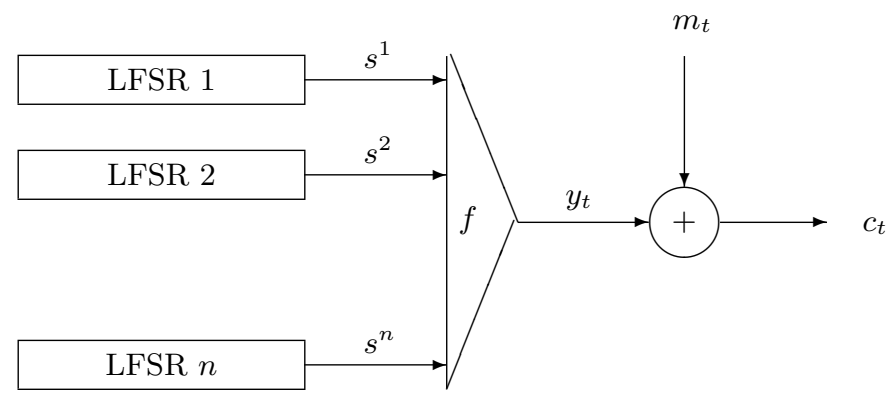

Fig. 1. Additive stream cipher using a combination generator

The other very important aspect is that the designs are often secret and contrary to block ciphers, generally no public evaluation is possible. Although such stream ciphers may be vulnerable to some attacks [1176], cryptanalysis becomes much harder when the algorithm is unknown. During World War II, US cryptanalysts had to face this problem with the Japanese PURPLE machine [8]: they reconstructed it before cryptanalysing it. This paper presents a similar approach and a reconstruction technique of stream ciphers allowing, from ciphertexts only, complete recovering of the unknown algorithm. By algebraic and statistical results, all the cryptographic primitives constituting the system (the LFSR characteristics and the combining function) can be recovered. After this reconstruction step, the LFSR initializations can be found by classical correlation attacks 11761 .

The reconstruction has been conducted on the following basis and assumptions:

- The system is a combination generator. Most practical designs use combining functions with up to 5 or 7 variables (i.e., registers). In this paper we will only consider additive stream ciphers but generalization to other combining functions can be envisaged with suitable modifications.

- We use only ciphertexts, possibly generated from different secret keys. Each of them, however, must be of a realistic length.

- We know the plaintext encoding (or at least some of its statistical parameters) and the linguistic group of the plaintext language.

- We accept very long computing time since work is done only once (and for all) and as long as it remains far lower than the life of the algorithm itself.

This paper is organized as follows. Section 2 presents the theoretical tools we use in the reconstruction. In Section 3, we show how to recover the LFSRs and we give some simulation results. We precisely analyze the complexity of this attack and we estimate the number of required ciphertext bits. Section 4 focuses on the combining function recovering. 


\section{Theoretical Background}

\subsection{Linear Feedback Shift Register Sequences}

A linear feedback shift register of length $L$ is characterized by a univariate polynomial $P$ over $\mathbf{F}_{2}$ of degree $L$, called the feedback polynomial, $P(x)=$ $1+\sum_{i=1}^{L} p_{i} X^{i}$. It associates to any $L$-bit initialization $\left(s_{t}\right)_{1 \leq t \leq L}$ a sequence $\left(s_{t}\right)_{t>0}$ defined by the $L$-th order linear recurrence relation.

$$
s_{t+L}=\sum_{i=1}^{L} p_{i} s_{t+L-i}, \quad t \geq 0 .
$$

Most applications use a primitive feedback polynomial since this ensures that the periods of all sequences produced by the LFSR are maximal.

We now recall some well-known properties on LFSR sequences. In the following, $\mathcal{S}(P)$ denotes the set of all sequences produced by the LFSR with feedback polynomial $P$.

Proposition 1. 15,14 Let $P$ and $Q$ be two non constant polynomials over $\mathbf{F}_{2}$. Then we have

$-\left\{\left(u_{t}+v_{t}\right)_{t>0}, u \in \mathcal{S}(P), v \in \mathcal{S}(Q)\right\}=\mathcal{S}(R)$ where $R$ is the least common multiple of $P$ and $Q$.

$-\left\{\left(u_{t} v_{t}\right)_{t>0}, u \in \mathcal{S}(P), v \in \mathcal{S}(Q)\right\}=\mathcal{S}(R)$ where $\operatorname{deg}(R) \leq \operatorname{deg}(P) \operatorname{deg}(Q)$. Equality holds if and only if at least one of the polynomials $P$ and $Q$ has only simple roots and all products $\alpha \beta$ are distinct for all $\alpha$ and $\beta$ such that $P(\alpha)=0$ and $Q(\beta)=0$ in a common splitting field. This condition is notably satisfied if $P$ and $Q$ have coprime orders.

Proposition 2. 9, Th. 8.53] Let $P$ and $Q$ be two non constant polynomials over $\mathbf{F}_{2}$. Then $\mathcal{S}(P)$ is a subset of $\mathcal{S}(Q)$ if and only if $P$ divides $Q$.

This proposition implies that if a sequence $s$ is generated by a LFSR with feedback polynomial $P$, then it satisfies the recurrence relations (or parity-check equations) corresponding to $P Q$ for any $Q \in \mathbf{F}_{2}[X]$.

For a given feedback polynomial $P$ of degree $L$, we focus on all recurrence relations corresponding to the multiples of $P$ of weight $d$, where $d$ is small. A similar approach is used in fast correlation attacks [1117]. The following formula (see e.g. [1]) provides an approximation of the average number $m(d)$ of multiples $Q$ of $P$ which have weight $d$ and degree at most $D, Q(X)=1+$ $\sum_{j=1}^{d-1} X^{i_{j}}$ :

$$
m(d) \simeq \frac{D^{d-1}}{(d-1) ! 2^{L}}
$$




\subsection{Boolean Functions for Stream Ciphers}

A Boolean function with $n$ variables is a function from the set of $n$-bit words, $\mathbf{F}_{2}^{n}$, into $\mathbf{F}_{2}$. Such a function can be expressed as a unique polynomial in $x_{1}, \ldots$, $x_{n}$, called its Algebraic Normal Form (ANF):

$$
f\left(x_{1}, \ldots, x_{n}\right)=\sum_{u \in \mathbf{F}_{2}^{n}} a_{u} x^{u}, a_{u} \in \mathbf{F}_{2}
$$

where $u=\left(u_{1}, \ldots, u_{n}\right)$ and $x^{u}=x_{1}^{u_{1}} x_{2}^{u_{2}} \ldots x_{n}^{u_{n}}$. The coefficients $a_{u}$ of the ANF can be obtained from the Möbius transform of $f$ [10]:

$$
a_{u}=\bigoplus_{x \preceq u} f(x)
$$

where $\oplus$ denotes the addition over $\mathbf{F}_{2}$ and $\alpha \preceq \beta$ describes the partial ordering on the Boolean lattice. This means that $\alpha \preceq \beta$ if and only if $\alpha_{i} \leq \beta_{i}$ for all $1 \leq i \leq n$.

The Walsh-Hadamard transform of a Boolean function $f$ refers to the Fourier transform of the corresponding sign function, $x \mapsto(-1)^{f(x)}$ :

$$
\forall u \in \mathbf{F}_{2}^{n}, \quad \widehat{\chi}_{f}(u)=\sum_{x \in \mathbf{F}_{2}^{n}}(-1)^{f(x)}(-1)^{u \cdot x}
$$

where $u \cdot x$ denotes the usual scalar product. The Walsh coefficient $\widehat{\chi}_{f}(u)$ then estimates the Hamming distance between $f$ and the affine function $u \cdot x+\varepsilon$, $\varepsilon \in \mathbf{F}_{2}$, both seen as Reed-Muller codewords [10].

A Boolean function is obviously completely characterized by its Walsh spectrum. The coefficients of the algebraic normal form of $f$ can then be computed from its Walsh coefficients as follows.

Proposition 3. Let $f$ be a Boolean function with $n$ variables and let $\left(a_{u}\right)_{u \in \mathbf{F}_{2}^{n}}$ denote the coefficients of its algebraic normal form, i.e.,

$$
f\left(x_{1}, \ldots, x_{n}\right)=\sum_{u \in \mathbf{F}_{2}^{n}} a_{u} x^{u}
$$

Then we have, for all $u \in \mathbf{F}_{2}^{n}, a_{u}=2^{w t(u)-1}\left(1-\frac{1}{2^{n}} \sum_{v \preceq \bar{u}} \widehat{\chi}_{f}(v)\right) \bmod 2$ where $\bar{u}$ denotes the bitwise completion to 1 and wt(u) is the Hamming weight of $u$, i.e., the number of its non-zero components.

Proof. From Equation (2) we have for any $u \in \mathbf{F}_{2}^{n}$

$$
\begin{aligned}
a_{u} & =\sum_{x \preceq u} f(x) \bmod 2=\sum_{x \preceq u} \frac{1}{2}\left(1-(-1)^{f(x)}\right) \bmod 2 \\
& =2^{w t(u)-1}-\frac{1}{2} \sum_{x \preceq u}(-1)^{f(x)} \bmod 2
\end{aligned}
$$


Since the normalized Fourier transform is involutive, we have

$$
\forall x \in \mathbf{F}_{2}^{n}, \quad(-1)^{f(x)}=2^{-n} \sum_{v \in \mathbf{F}_{2}^{n}} \widehat{\chi}_{f}(v)(-1)^{v \cdot x} .
$$

By combining these relations, we deduce that

$$
\begin{aligned}
a_{u} & =2^{w t(u)-1}-2^{-n-1} \sum_{x \preceq u} \sum_{v \in \mathbf{F}_{2}^{n}} \widehat{\chi}_{f}(v)(-1)^{v \cdot x} \bmod 2 \\
& =2^{w t(u)-1}-2^{-n-1} \sum_{v \in \mathbf{F}_{2}^{n}} \widehat{\chi}_{f}(v)\left(\sum_{x \preceq u}(-1)^{v \cdot x}\right) \bmod 2 .
\end{aligned}
$$

The set $E_{u}=\left\{x \in \mathbf{F}_{2}^{n}, x \preceq u\right\}$ is a linear subspace of $\mathbf{F}_{2}^{n}$ of dimension $w t(u)$. Its orthogonal, $E_{u}^{\perp}$, satisfies $E_{u}^{\perp}=E_{\bar{u}}$. It follows that

$$
\sum_{x \preceq u}(-1)^{v \cdot x}= \begin{cases}2^{w t(u)} & \text { if } v \in E_{\bar{u}}, \\ 0 & \text { otherwise. }\end{cases}
$$

We then obtain that, for all $u \in \mathbf{F}_{2}^{n}$,

$$
a_{u}=2^{w t(u)-1}-2^{-n-1+w t(u)} \sum_{v \preceq \bar{u}} \widehat{\chi}_{f}(v) \bmod 2 .
$$

This proposition will be used in the attack for recovering the algebraic normal form of the combining function.

It is well-known that a combining function must fulfill some criteria to yield a cryptographically secure combination generator (see e.g. [3]). Most notably, combination generators are vulnerable to "divide-and-conquer" attacks, called correlation attacks [17. These techniques fail when the combining function has a high correlation-immunity order [16].

Definition 1. A Boolean function is t-th order correlation-immune if the probability distribution of its output is unaltered when any $t$ input variables are fixed.

This property equivalently asserts that the output of $f$ is statistically independent of any linear combination of $t$ input variables. Correlation-immunity can be characterized by the Walsh spectrum of the function [18]: $f$ is $t$-th order correlation-immune if and only if

$$
\forall u \in \mathbf{F}_{2}^{n}, 1 \leq w t(u) \leq t, \quad \widehat{\chi}_{f}(u)=0
$$

Since any $t$-th order correlation-immune function is $k$-th order correlation-immune for any $k \leq t$, we call correlation-immunity order of a function $f$ the highest integer $t$ such that $f$ is $t$-th order correlation-immune. Note that the correlationimmunity order of a function with $n$ variables can not exceed $(n-1)$. This comes from Parseval's relation:

$$
\sum_{u \in \mathbf{F}_{2}^{n}}\left(\widehat{\chi}_{f}(u)\right)^{2}=2^{2 n}
$$


This equality also points out the existence of a trade-off between the correlationimmunity order and the nonlinearity of a function.

\section{Recovering the LFSRs}

We now show how the key-stream generator depicted in Figure 1 can be reconstructed from the knowledge of some ciphertext bits.

In the rest of the paper we use the following notation. $n$ denotes the number of constituent LFSRs. $L_{i}$ and $P_{i}$ denote the length and the feedback polynomial of the $i$-th LFSR and $s^{i}$ refers to the generated sequence. The sequences $y, m$ and $c$ respectively correspond to the key-stream, to the plaintext and to the ciphertext. When dealing bitwise, we use $t$ as index time.

The plaintext is assumed to be the output of a binary memoryless source with $P\left[m_{t}=0\right]=p_{0} \neq \frac{1}{2}$. All commonly used coding scheme (ASCII, Murray, CCITTx ...) satisfy this hypothesis. Moreover, the value of $p_{0}$ is supposed to be known. Practical values of $p_{0}$ are usually greater than 0.6 .

The first step of the reconstruction consists in recovering the feedback polynomials of the constituent LFSRs.

\subsection{Statistical Model}

We first point out that the knowledge of a sequence $s$ which is correlated with the ciphertext sequence provides some information on the feedback polynomials of the constituent LFSRs.

Proposition 4. Let $s$ be a binary sequence. If $P\left[c_{t}=s_{t}\right] \neq 1 / 2$ then there exists a Boolean function $g$ with $n$ variables such that $s=g\left(s^{1}, \ldots, s^{n}\right)$. Moreover, we have $P\left[c_{t}=s_{t}\right]=1-p_{0}-p_{g}+2 p_{0} p_{g}$ where $p_{g}=P\left[f\left(x_{1}, \ldots, x_{n}\right)=g\left(x_{1}, \ldots, x_{n}\right)\right]$.

Proof. We obviously have

$$
\begin{aligned}
P\left[c_{t}=s_{t}\right] & =P\left[y_{t}=s_{t}\right] P\left[m_{t}=0\right]+P\left[y_{t}=s_{t} \oplus 1\right] P\left[m_{t}=1\right] \\
& =1-p_{0}-P\left[y_{t}=s_{t}\right]+2 p_{0} P\left[y_{t}=s_{t}\right] .
\end{aligned}
$$

By hypothesis, $p_{0} \neq 1 / 2$. Thus $P\left[c_{t}=s_{t}\right] \neq 1 / 2$ implies that $P\left[y_{t}=s_{t}\right] \neq 1 / 2$. Since $y=f\left(s^{1}, \ldots, s^{n}\right)$, the sequences $y$ and $s$ are statistically independent if $s$ is statistically independent of $\left(s^{1}, \ldots, s^{n}\right)$. It follows that $P\left[y_{t}=s_{t}\right]=1 / 2$ unless $s=g\left(s^{1}, \ldots, s^{n}\right)$ for some Boolean function $g$. In this case, we have

$$
P\left[y_{t}=c_{t}\right]=P\left[f\left(x_{1}, \ldots, x_{n}\right)=g\left(x_{1}, \ldots, x_{n}\right)\right] .
$$

Note that some variables may not appear in the algebraic normal form of $g$.

If $s$ is such that $P\left[c_{t}=s_{t}\right] \neq 1 / 2$ we deduce from the previous proposition and from Proposition 1 that the feedback polynomial of $s$ is related to the feedback polynomials $P_{1}, \ldots, P_{n}$. 
Corollary 1. Let $\mathcal{S}(Q)$ denote the set of all sequences generated by $Q \in \mathbf{F}_{2}[X]$. If there exists $s \in \mathcal{S}(Q)$ such that $P\left[c_{t}=s_{t}\right] \neq 1 / 2$, then there exists a divisor $Q^{\prime}$ of $Q$ and a Boolean function $g$ such that $Q^{\prime}$ is derived from $P_{1}, \ldots, P_{n}$ and from $g$ as described in Proposition 1 .

This result leads to the following algorithm for recovering some information on $P_{1}, \ldots, P_{n}$. Let $\mathcal{Q}$ be a subset of $\mathbf{F}_{2}[X]$. For each $Q \in \mathcal{Q}$, we determine whether $\mathcal{S}(Q)$ contains a sequence which is correlated with the ciphertext. If such a sequence exists, $Q$ provides some information on $P_{1}, \ldots, P_{n}$. We here choose for $\mathcal{Q}$ the set of all polynomials of $\mathbf{F}_{2}[X]$ of weight $d$ and of degree at most $D$ having the following form $Q(X)=1+\sum_{j=1}^{d-1} X^{i_{j}}$. Recall that the degree of the feedback polynomial of the product of two sequences $s^{i}$ and $s^{j}$ is usually much higher than the degree of the feedback polynomial of $s^{i}+s^{j}$. If the upper-bound $D$ on the degree of the examined polynomials is well-chosen, the polynomials $Q$ detected by the algorithm then correspond to the case where the combining function $g$ is linear. For $g(x)=u \cdot x$, any feedback polynomial of $s=g\left(s^{1}, \ldots, s^{n}\right)$ is a multiple of $\operatorname{lcm}_{i \in \operatorname{supp}(u)} P_{i}$ where $\operatorname{supp}(u)=\left\{i, u_{i}=1\right\}$. Since all feedback polynomials are usually primitive, we have $\operatorname{lcm}_{i \in \operatorname{supp}(u)} P_{i}=\prod_{i \in \operatorname{supp}(u)} P_{i}$ in most practical situations. Moreover, we have

$$
P\left[c_{t}=s_{t}\right]=\frac{1}{2}+\frac{\left(2 p_{0}-1\right)}{2^{n+1}} \widehat{\chi}_{f}(u) .
$$

Example 1. We consider the combination generator described by Geffe [4]. It consists of three LFSRs combined by the Boolean function $f\left(x_{1}, x_{2}, x_{3}\right)=x_{1} x_{2}+$ $x_{2} x_{3}+x_{1}$. Assume that the feedback polynomials of the constituent LFSRs are randomly chosen primitive polynomials and that their lengths are respectively $L_{1}=15, L_{2}=17$ and $L_{3}=23$. Let $c$ be the ciphertext sequence obtained by adding the output of Geffe generator to a plaintext with $p_{0} \neq 0.5$. Let $\mathcal{Q}$ be the set of all polynomials of weight 4 and of degree at most 10000 . For all $Q \in \mathcal{Q}$, we determine whether $\mathcal{S}(Q)$ contains a sequence which is correlated with $c$. We deduce from Formula (11) that, for a randomly chosen polynomial $P$ of degree $L$, $\mathcal{Q}$ contains a multiple of $P$ of weight 4 if $L \leq 37$. Our algorithm is then expected to detect multiples of $P_{1}, P_{2}, P_{3}$ and $P_{1} P_{2}$. Note that $P_{2}$ can not be detected by the algorithm since the Walsh coefficient $\widehat{\chi}_{f}(0,1,0)$ vanishes.

A simple method for determining whether $\mathcal{S}(Q)$ contains a sequence which is correlated with $c$ consists in computing the parity-check equation corresponding to $Q$ for the ciphertext bits. The efficiency of this procedure strongly depends on the weight of $Q$.

Theorem 1. Let $Q$ be a polynomial in $\mathbf{F}_{2}[X]$ of weight $d$ having the following form $Q(X)=1+\sum_{j=1}^{d-1} X^{i_{j}}$ with $i_{1}<i_{2}<\ldots<i_{d-1}$. For a given ciphertext subsequence $\left(c_{t}\right)_{t<N}$ we consider the binary sequence $\left(z_{t}\right)_{i_{d-1} \leq t<N}$ defined by

$$
z_{t}=c_{t} \oplus \bigoplus_{j=1}^{d-1} c_{t-i_{j}}
$$


Then the random variable $Z=\sum_{t=i_{d-1}}^{N-1}(-1)^{z_{t}}$ has a Gaussian distribution with mean value $M= \pm\left(N-i_{d-1}\right)(2 \varepsilon)^{d}$ and with variance $\sigma^{2}=\left(N-i_{d-1}\right)\left(1-(2 \varepsilon)^{2 d}\right)$ where $\varepsilon=\max _{s \in \mathcal{S}(Q)}\left|P\left[c_{t}=s_{t}\right]-\frac{1}{2}\right|$.

Proof. Let $s \in \mathcal{S}(Q)$ be such that $\left|P\left[c_{t}=s_{t}\right]-\frac{1}{2}\right|$ is maximal. Let $p=P\left[c_{t}=s_{t}\right]$. For all $t$, we decompose $c_{t}$ as $c_{t}=s_{t} \oplus e_{t}$ where $P\left[e_{t}=1\right]=1-p$. Then we have

$$
P\left[z_{t}=1\right]=P\left[c_{t} \oplus \bigoplus_{j=1}^{d-1} c_{t-i_{j}}=1\right]=P\left[e_{t} \oplus \bigoplus_{j=1}^{d-1} e_{t-i_{j}}=1\right]
$$

since $s$ satisfies the recurrence relation because $s \in \mathcal{S}(Q)$. This implies that $z_{t}=1$ if and only if the number of indexes $i \in\left\{t, t-i_{1}, \ldots, t-i_{d-1}\right\}$ such that $e_{i}=1$ is odd. Therefore we have

$$
\begin{aligned}
P\left[z_{t}=1\right] & =\sum_{\ell=0, \ell \text { odd }}^{d}\left(\begin{array}{l}
d \\
\ell
\end{array}\right)(1-p)^{\ell} p^{d-\ell} \\
& =\frac{1}{2}\left[\sum_{\ell=0}^{d}\left(\begin{array}{l}
d \\
\ell
\end{array}\right)(1-p)^{\ell} p^{d-\ell}-\sum_{\ell=0}^{d}\left(\begin{array}{l}
d \\
\ell
\end{array}\right)(p-1)^{\ell} p^{d-\ell}\right] \\
& =\frac{1}{2}\left[1-(2 p-1)^{d}\right] .
\end{aligned}
$$

The random variable $Z$ can now be expressed as $Z=\left(N-i_{d-1}\right)-2 \sum_{t=i_{d-1}}^{N} z_{t}$. All random variables $z_{t}$ are independent and identically distributed. Due to the central limit theorem [2], the random variable $\sum_{t=i_{d-1}}^{N} z_{t}$ for large values of $\left(N-i_{d-1}\right)$ can be assumed to have a Gaussian distribution with mean value $\left(N-i_{d-1}\right) P\left[z_{t}=1\right]$ and variance $\left(N-i_{d-1}\right) P\left[z_{t}=1\right] P\left[z_{t}=0\right]$. It follows that $Z$ has a Gaussian distribution with mean value

$$
M=\left(N-i_{d-1}\right)\left(1-2 P\left[z_{t}=1\right]\right)=\left(N-i_{d-1}\right)(2 p-1)^{d}
$$

and with variance

$$
\begin{aligned}
\sigma^{2} & =4\left(N-i_{d-1}\right) P\left[z_{t}=1\right] P\left[z_{t}=0\right]=\left(N-i_{d-1}\right)\left(1-(2 p-1)^{d}\right)\left(1+(2 p-1)^{d}\right) \\
& =\left(N-i_{d-1}\right)\left(1-(2 p-1)^{2 d}\right)
\end{aligned}
$$

If all sequences in $\mathcal{S}(Q)$ are statistically independent of $c, Z$ has Gaussian distribution with mean value 0 and variance $\left(N-i_{d-1}\right)$ since $\varepsilon=0$ in this case.

We now want to distinguish between two hypotheses:

$-\mathcal{H}_{0}:$ for all $s \in \mathcal{S}(Q), P\left[c_{t}=s_{t}\right]=\frac{1}{2}$.

$-\mathcal{H}_{1}$ : there exists $s \in \mathcal{S}(Q)$ such that $P\left[s_{t}=c_{t}\right] \neq \frac{1}{2}$.

We use a decision threshold $T, T>0$, for discriminating hypotheses $\mathcal{H}_{0}$ and $\mathcal{H}_{1}$. If $|Z|<T, \mathcal{H}_{0}$ is kept; if $|Z| \geq T, \mathcal{H}_{1}$ is accepted. The minimum number of required ciphertext bits, $N$, depends on the number of wrong decisions that 
we allow. This number corresponds to the probability for a false alarm, $P_{f}=$ $P\left[|Z| \geq T \mid \mathcal{H}_{0}\right]$. The decision threshold is determined by the probability for a non-detection, $P_{n}=P\left[|Z|<T \mid \mathcal{H}_{1}\right]$. Let $\Phi$ denotes the normal distribution function,

$$
\Phi(x)=\frac{1}{\sqrt{2 \pi}} \int_{-\infty}^{x} \exp \left(-\frac{x^{2}}{2}\right) d x .
$$

Then we have

$$
P_{f}=P\left[|Z| \geq T \mid \mathcal{H}_{0}\right]=2 \Phi\left(\frac{-T}{\sqrt{N-i_{d-1}}}\right) .
$$

Similarly the probability for a non-detection is given by

$$
\begin{aligned}
P_{n} & =P\left[|Z|<T \mid \mathcal{H}_{1}\right]=\frac{1}{\sqrt{2 \pi}} \int_{\frac{-T-M}{\sigma}}^{\frac{T-M}{\sigma}} \exp \left(-\frac{x^{2}}{2}\right) d x \\
& =\Phi\left(\frac{T-M}{\sigma}\right)-\Phi\left(\frac{-T-M}{\sigma}\right)=\Phi\left(\frac{T-|M|}{\sigma}\right)-\Phi\left(\frac{-T-|M|}{\sigma}\right)
\end{aligned}
$$

since $M$ is not necessarily positive. In most cases, $\Phi\left(\frac{-T-|M|}{\sigma}\right)$ is much smaller than $P_{n}$ and than $\Phi\left(\frac{T-|M|}{\sigma}\right)$. Then this latter will approximate $P_{n}$. The predetermined value of $P_{n}$ fixes the choice for the threshold:

$$
T=|M|+\Phi^{-1}\left(P_{n}\right) \sigma=\left(N-i_{d-1}\right)(2 \varepsilon)^{d}+\Phi^{-1}\left(P_{n}\right) \sqrt{\left(N-i_{d-1}\right)\left(1-(2 \varepsilon)^{2 d}\right)} .
$$

Similarly, the predetermined probability for a false alarm gives the minimum value of $\left(N-i_{d-1}\right)$ :

$$
N-i_{d-1}=\left(\frac{T}{\Phi^{-1}\left(1-\frac{P_{f}}{2}\right)}\right)^{2} .
$$

After different attempts to tune up the best values for $P_{f}$ and $P_{n}$, we choose $P_{f}=2^{-20}$ and $P_{n}=10^{-3}$. In practical situations the known ciphertext sequence does not consist of a large number of consecutive bits. The attacker has access to some ciphertext blocks of reasonable lengths. These ciphertexts may be produced with different keys, i.e., with different LFSR initializations. Theorem 1 can nevertheless be adapted to this more realistic situation.

Corollary 2. Let $Q$ be a polynomial in $\mathbf{F}_{2}[X]$ of weight d having the following form $Q(X)=1+\sum_{j=1}^{d-1} X^{i_{j}}$ with $i_{1}<i_{2}<\ldots<i_{d-1}$. For $n_{c}$ ciphertexts $c^{k}, 1 \leq k \leq n_{c}$, of respective lengths $L C(k)$, we consider the binary sequence $\left(z_{t}^{k}\right)_{i_{d-1} \leq t<L C(k), 1 \leq k \leq n_{c}}$ defined by

$$
z_{t}^{k}=c_{t}^{k} \oplus \bigoplus_{j=1}^{d-1} c_{t-i_{j}}^{k}
$$


Then the random variable

$$
Z=\sum_{k=1}^{n_{c}} \sum_{t=i_{d-1}}^{L C(k)-1}(-1)^{z_{t}^{k}}
$$

has a Gaussian distribution with mean value

$$
M= \pm(2 \varepsilon)^{d} \sum_{k=1}^{n_{c}}\left(L C(k)-i_{d-1}\right)
$$

and with variance

$$
\sigma^{2}=\left(1-(2 \varepsilon)^{2 d}\right) \sum_{k=1}^{n_{c}}\left(L C(k)-i_{d-1}\right)
$$

where $\varepsilon=\max _{s \in \mathcal{S}(Q)}\left|P\left[c_{t}=s_{t}\right]-\frac{1}{2}\right|$.

The following algorithm then examines all polynomials of degree at most $D$ and of weight $d$, and it detects all polynomials $Q$ in this set such that there exists $s \in \mathcal{S}(Q)$ with $\left|P\left[s_{t}=c_{t}\right]-1 / 2\right| \geq \varepsilon_{\min }$.

\section{Algorithm}

For each $(d-1)$-tuples $\left(i_{1}, \ldots, i_{d-1}\right)$ such that $0<i_{1}<\ldots<i_{d-1}<D$

$$
\begin{aligned}
& N \leftarrow \sum_{k=1}^{n_{c}}\left(L C(k)-i_{d-1}\right) . \\
& T \leftarrow N\left(2 \varepsilon_{\text {min }}\right)^{d}-3 \sqrt{N\left(1-\left(2 \varepsilon_{\text {min }}\right)^{2 d}\right)} . \\
& Z \leftarrow 0 .
\end{aligned}
$$

For each ciphertext block $\left(c_{t}^{k}\right)_{0 \leq t<L C(k)}$ where $L C(k)>i_{d-1}$

for each $t$ from $i_{d-1}$ to $L C(k)-1$

$$
\begin{aligned}
& z \leftarrow c_{t}^{k} \oplus \bigoplus_{j=1}^{d-1} c_{t-i_{j}}^{k} . \\
& Z \leftarrow Z+(-1)^{z} .
\end{aligned}
$$

If $|Z| \geq T$, store $1+\sum_{j=1}^{d-1} X^{i_{j}}$ and the value of $Z$.

Some gcd computations on the obtained polynomials provide the primitive factors which are detected several times. These primitive factors are expected to be the feedback polynomials of the constituent LFSRs.

\subsection{Complexity Analysis}

We now discuss the choice of the input parameters $d, D$ and $\varepsilon_{\min }$.

Recall that we aim at recovering multiples of polynomials $\prod_{i \in T} P_{i}, T \subset$ $\{1, \ldots, n\}$ such that $\left|P\left[c_{t}=\bigoplus_{i \in T} s_{t}^{i}\right]-1 / 2\right| \geq \varepsilon_{\min }$. According to Formula (3), these subsets $T$ are characterized by

$$
\frac{\left|2 p_{0}-1\right|}{2^{n+1}}\left|\widehat{\chi}_{f}\left(1_{T}\right)\right| \geq \varepsilon_{\min }
$$


where the $i$-th component of $1_{T}$ equals 1 if and only if $i \in T$. It is well-known that all Walsh coefficients of a Boolean function $f$ with $n$ variables are divisible by 4 , unless $f$ has degree $n$. This case is here dismissed since such a function cannot be balanced. Choosing

$$
\varepsilon_{\min }=\frac{\left|2 p_{0}-1\right|}{2^{n-1}}
$$

then ensures to detect all polynomials $\prod_{i \in T} P_{i}$ such that $\widehat{\chi}_{f}\left(1_{T}\right) \neq 0$. In most practical situations, the number of variables $n$ does not exceed 7 .

We now assume that our search can be restricted to all products $\prod_{i \in T} P_{i}$ of degree at most $L_{\max }$. This means that we suppose that all feedback polynomials $P_{1}, \ldots, P_{n}$ can be recovered from all products $\prod_{i \in T} P_{i}$ such that $\widehat{\chi}_{f}\left(1_{T}\right) \neq 0$ and $\sum_{i \in T} L_{i} \leq L_{\max }$. Note that $L_{\max }$ should obviously be greater than the maximum length of all constituent LFSRs. A polynomial of degree $L_{\max }$ is then recovered by our algorithm if it divides at least one polynomial of weight $d$ and of degree at most $D$. We deduce from Formula (1) that the minimum possible value for $D$ is approximatively

$$
D=(d-1) ! \frac{1}{d-1} 2^{\frac{L_{\max }}{d-1}} .
$$

This also implies that the attack can only use ciphertext blocks of length at least $L C$ with

$$
L C \geq(d-1) ! \frac{1}{d-1} 2^{\frac{L_{\max }}{d-1}} .
$$

Moreover, we want the probability for a false alarm in the algorithm to be less than $2^{-20}$. This implies that $\left(\sum_{k=1}^{n_{c}} L C(k)\right)-n_{c} D \geq\left(\frac{T}{5}\right)^{2}$. By replacing $T$ by its value, we obtain the following condition

$$
N_{t}-n_{c} D \geq \frac{1}{25}\left(\left(N_{t}-n_{c} D\right)\left(2 \varepsilon_{\min }\right)^{d}-3 \sqrt{\left(N_{t}-n_{c} D\right)\left(1-\left(2 \varepsilon_{\min }\right)^{2 d}\right)}\right)^{2}
$$

where $N_{t}=\sum_{k=1}^{n_{c}} L C(k)$ is the total ciphertext length. We deduce that

$$
N_{t}-n_{c} D \geq \frac{\left(5+3 \sqrt{1-\left(2 \varepsilon_{\min }\right)^{2 d}}\right)^{2}}{\left(2 \varepsilon_{\min }\right)^{2 d}} .
$$

It finally follows that the total ciphertext length should satisfy

$$
N_{t} \geq n_{c}(d-1) ! \frac{1}{d-1} 2^{\frac{L_{\max }}{d-1}}+\frac{\left(5+3 \sqrt{1-\left(2 \varepsilon_{\min }\right)^{2 d}}\right)^{2}}{\left(2 \varepsilon_{\min }\right)^{2 d}} .
$$

This value is minimal if $n_{c}=1$, i.e., if all known ciphertext bits are consecutive. In this case, the minimum length of the ciphertext sequence required by the reconstruction is

$$
N_{t}=\min _{d}\left[(d-1) !^{\frac{1}{d-1}} 2^{\frac{L_{\max }}{d-1}}+\frac{\left(5+3 \sqrt{1-\left(2 \varepsilon_{\min }\right)^{2 d}}\right)^{2}}{\left(2 \varepsilon_{\min }\right)^{2 d}}\right] .
$$


This formula points out that the optimal value of $d$ increases with $L_{\max }$. Figure 2 shows how $N_{t}$ and the optimal value of $d$ vary with $\varepsilon_{\min }$, for $L_{\max }=70$.

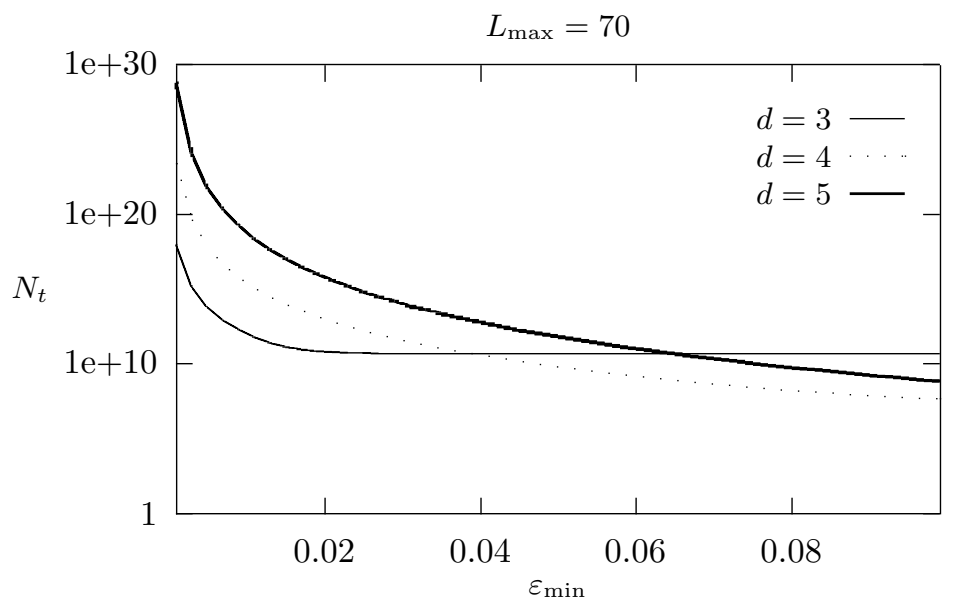

Fig. 2. Minimum ciphertext length required for $L_{\max }=70$

In most practical situations, all ciphertext blocks have roughly the same length $L C$. The number $n_{c}$ of such ciphertext blocks required by the reconstruction is then

$$
n_{c} \geq \frac{\left(5+3 \sqrt{1-\left(2 \varepsilon_{\min }\right)^{2 d}}\right)^{2}}{\left(2 \varepsilon_{\min }\right)^{2 d}\left(L C-(d-1) ! \frac{1}{d-1} 2^{\frac{L_{\max }}{d-1}}\right)} .
$$

We then use the algorithm with the value of $d$ which minimizes this formula.

The number of operations performed by the algorithm is roughly

$$
\frac{D^{d-1}}{(d-1) !} d\left(N_{t}-n_{c} D\right) \text {. }
$$

Using equations (5) and (8), we obtain the following complexity

$$
\frac{d 2^{L_{\max }}\left(5+3 \sqrt{1-\left(2 \varepsilon_{\min }\right)^{2 d}}\right)^{2}}{\left(2 \varepsilon_{\min }\right)^{2 d}} .
$$

Another method for recovering the feedback polynomials of the LFSRs consists in examining all polynomials of degree at most $L_{\max }$ and in computing the corresponding parity-check equations on the ciphertext sequence. A similar analysis applies to this attack. We here have to choose $D=L_{\max }$ and $d \simeq L_{\max } / 2$ since the average weight of a polynomial of degree $L_{\max }$ is roughly $L_{\max } / 2$. With 
these parameters, Formula (7) provides the minimum ciphertext length required by this second attack:

$$
N_{t}^{\prime}=L_{\max }+\frac{\left(5+3 \sqrt{1-\left(2 \varepsilon_{\min }\right)^{L_{\max }}}\right)^{2}}{\left(2 \varepsilon_{\min }\right)^{L_{\max }}} .
$$

We easily see that this number is much larger that the number of ciphertext bits required by our attack (see Formula (8)). Moreover, the number of operations performed by this second attack is roughly

$$
\frac{d 2^{L_{\max }}\left(5+3 \sqrt{1-\left(2 \varepsilon_{\min }\right)^{L_{\max }}}\right)^{2}}{2\left(2 \varepsilon_{\min }\right)^{L_{\max }}} .
$$

Our attack is then much more efficient than the enumeration of all polynomials of degree $L_{\max }$.

\subsection{Simulation Results}

We consider the following toy example of combination generator. Three LFSRs are combined by the majority function $f\left(x_{1}, x_{2}, x_{3}\right)=x_{1} x_{2}+x_{1} x_{3}+x_{2} x_{3}$. The feedback polynomials are respectively

$$
\begin{aligned}
& P_{1}(x)=1+x^{2}+x^{4}+x^{5}+x^{6}+x^{8}+x^{9}+x^{10}+x^{11}+x^{13}+x^{15} \\
& P_{2}(x)=1+x^{2}+x^{4}+x^{5}+x^{6}+x^{8}+x^{9}+x^{10}+x^{11}+x^{13}+x^{14}+x^{15}+x^{17} \\
& P_{3}(x)=1+x+x^{7}+x^{8}+x^{10}+x^{12}+x^{15}+x^{16}+x^{17}+x^{20}+x^{21}+x^{22}+x^{23}
\end{aligned}
$$

The output of this combination generator is used for encrypting a plaintext with $p_{0}=0.70$. We take $\varepsilon_{\min }=0.1$; this value corresponds to Formula (4) with $n=3$. We applied our algorithm with parameters $D=3,620$ and $d=3$ (which is the optimal value for these parameters). We used 170 ciphertext blocks of length 10,000 (i.e., around 1,200 ASCII characters). Note that Formula (10) gives $n_{c}=157$. Exactly 263 trinomials have been detected by our algorithm. All of these trinomials are divisible by one of the feedback polynomials. This means that the effective probability for a false alarm is zero. Moreover, all multiples of $P_{1}, P_{2}$ and $P_{3}$ of degree at most 3,620 have been detected (see Table 1 ). This

Table 1. Detected polynomials for the toy example

\begin{tabular}{|c|c|c|c|c|c|}
\hline & $d$ & $P_{1}$ & $P_{2}$ & $P_{3}$ & Total \\
\hline \hline Nb. of detected polynomials & 3 & 208 & 53 & 2 & 263 \\
\hline Exact nb. of multiples & 3 & 208 & 53 & 2 & 263 \\
\hline
\end{tabular}

simulation required roughly one week on a DEC alpha workstation at $433 \mathrm{MHz}$. 
We also checked our attack on the same example where $P_{1}$ was replaced by $1+x+x^{3}+x^{6}+x^{7}+x^{8}+x^{13}+x^{16}+x^{19}+x^{20}+x^{25}+x^{26}+x^{27}+x^{28}+x^{29}+x^{31}+x^{33}$.

We here used 6,109 ciphertext blocks of length 10,000. The optimal parameters are here $D=5,910$ and $d=4$. For these values, all multiples of $P_{1}, P_{2}$ and $P_{3}$, of weight 4 and degree at most $D$ have been detected.

\section{Recovering of the Combining Function}

A method for recovering the combining function was developed in [12] but it requires the knowledge of all LFSR initializations. Moreover, this technique relies on Siegenthaler's correlation attack; its complexity is then exponential in the lengths of the constituent LFSRs. We now show how to bypass these limitations and to practically reconstruct the combining function.

The number of variables of the combining function is derived from the previous step of our attack. Moreover, the previous analysis also provides an estimation of some Walsh coefficients of the combining function. Suppose that some multiples of weight $d$ of $\prod_{i \in T} P_{i}, T \subset\{1, \ldots, n\}$, have been detected by our algorithm. For any such multiple, the mean value of the estimator $Z$ equals $N(2 p-1)^{d}$, where $p=P\left[c_{t}=s_{t}\right]$ with $s=g\left(s^{1}, \ldots, s^{n}\right)$ and $g(x)=1_{T} \cdot x$. The values of $Z$ obtained for all detected multiples of $\prod_{i \in T} P_{i}$ therefore provides an estimation of probability $p$. Using Formula (3), we can then compute the value of the corresponding Walsh coefficient, $\widehat{\chi}_{f}\left(1_{T}\right)$. This value is rounded to the closest multiple of 4 , since all the Walsh coefficients are divisible by 4 for balanced functions.

If $\prod_{i \in T} P_{i}$ has degree $L$ greater than $L_{\max }$, no multiple was detected by the algorithm. We then choose a higher value of $d$ satisfying

$$
(d-1) ! \frac{1}{d-1} 2^{\frac{L}{d-1}} \leq L C .
$$

We then compute all multiples of $\prod_{i \in T} P_{i}$ of weight $d$ and degree at most $L C$, and the corresponding values of $Z$. We deduce the involved Walsh coefficient as previously seen.

Example 2. In the toy example, the values of the estimator $Z$ obtained for each multiple of weight 3 of $P_{1}$ provide

$$
P\left[c_{t}=s_{t}\right]=0.6003
$$

Formula (3) gives the approximation: $\widehat{\chi_{f}}(1,0,0)=4.01$. Similarly, we obtain the following information during the first step:

$$
\widehat{\chi_{f}}(0,1,0)=\widehat{\chi_{f}}(1,0,0)=4 \quad \widehat{\chi_{f}}(0,0,0)=0 .
$$


For each detected $P_{i}$ we compute some multiples of weight 5 and of degree at most 10,000 for each product $P_{i} P_{j}$. Although all of these products were potentially detectable, no one was detected; we then deduce that

$$
\widehat{\chi_{f}}(1,1,0)=\widehat{\chi_{f}}(1,0,1)=\widehat{\chi_{f}}(0,1,1)=0 .
$$

Similar simulations for $d=7$ allow to find the remaining coefficient:

$$
\widehat{\chi_{f}}(1,1,1)=-4 \text {. }
$$

Acknowledgments. This paper is dedicated to Colonel Max Mayneris, a very enthusiastic signal officer, who very early understood the importance of working on such subjects. We will have an everlasting thought for his influence. We hope he now enjoys his retirement in his beloved Pyrénées mountains.

\section{References}

1. A. Canteaut and M. Trabbia. Improved fast correlation attacks using parity-check equations of weight 4 and 5. In Advances in Cryptology - EUROCRYPT 2000, Lecture Notes in Computer Science. Springer-Verlag, 2000. To appear.

2. W. Feller. An Introduction to Probability Theory. Wiley, 1966.

3. E. Filiol and C. Fontaine. Highly nonlinear balanced Boolean functions with a good correlation-immunity. In Advances in Cryptology - EUROCRYPT'98, number 1403 in Lecture Notes in Computer Science, pages 475-488. Springer-Verlag, 1998.

4. P.R. Geffe. How to protect data with ciphers that are really hard to break. Electronics, pages 99-101, 1973.

5. T. Herlestam. On functions of linear shift register sequences. In F. Pichler, editor, Advances in Cryptology - EUROCRYPT '85, number 219 in Lecture Notes in Computer Science, pages 119-129. Springer-Verlag, 1986.

6. T. Johansson and F. Jönsson. Fast correlation attacks based on turbo code techniques. In Advances in Cryptology - CRYPTO'99, number 1666 in Lecture Notes in Computer Science, pages 181-197. Springer-Verlag, 1999.

7. T. Johansson and F. Jönsson. Improved fast correlation attack on stream ciphers via convolutional codes. In Advances in Cryptology - EUROCRYPT'99, number 1592 in Lecture Notes in Computer Science, pages 347-362. Springer-Verlag, 1999.

8. D. Kahn. The Codebreakers: The Story of Secret Writings. Macmillan Publishing Co, 1967.

9. R. Lidl and H. Niederreiter. Finite fields. Cambridge University Press, 1983.

10. F.J. MacWilliams and N.J.A. Sloane. The theory of Error-correcting codes. NorthHolland, 1977.

11. W. Meier and O. Staffelbach. Fast correlation attack on certain stream ciphers. J. Cryptology, pages 159-176, 1989.

12. S. Palit and B. Roy. Cryptanalysis of LFSR-encrypted codes with unknown combining function. In ASIACRYPT'99, number 1716 in Lecture Notes in Computer Science. Springer-Verlag, 1999.

13. R.A. Rueppel. Analysis and Design of stream ciphers. Springer-Verlag, 1986.

14. R.A. Rueppel and O.J. Staffelbach. Products of linear recurring sequences with maximum complexity. IEEE Trans. Inform. Theory, 33(1):124-131, 1987. 
15. E.S. Selmer. Linear recurrence relations over finite fields. PhD thesis, University of Bergen, Norway, 1966.

16. T. Siegenthaler. Correlation-immunity of nonlinear combining functions for cryptographic applications. IEEE Trans. Inform. Theory, IT-30(5):776-780, 1984.

17. T. Siegenthaler. Decrypting a class of stream ciphers using ciphertext only. IEEE Trans. Computers, C-34(1):81-84, 1985.

18. G. Xiao and J.L. Massey. A spectral characterization of correlation-immune combining functions. IEEE Trans. Inform. Theory, IT-34(3):569-571, 1988. 